\title{
Model - 1
}

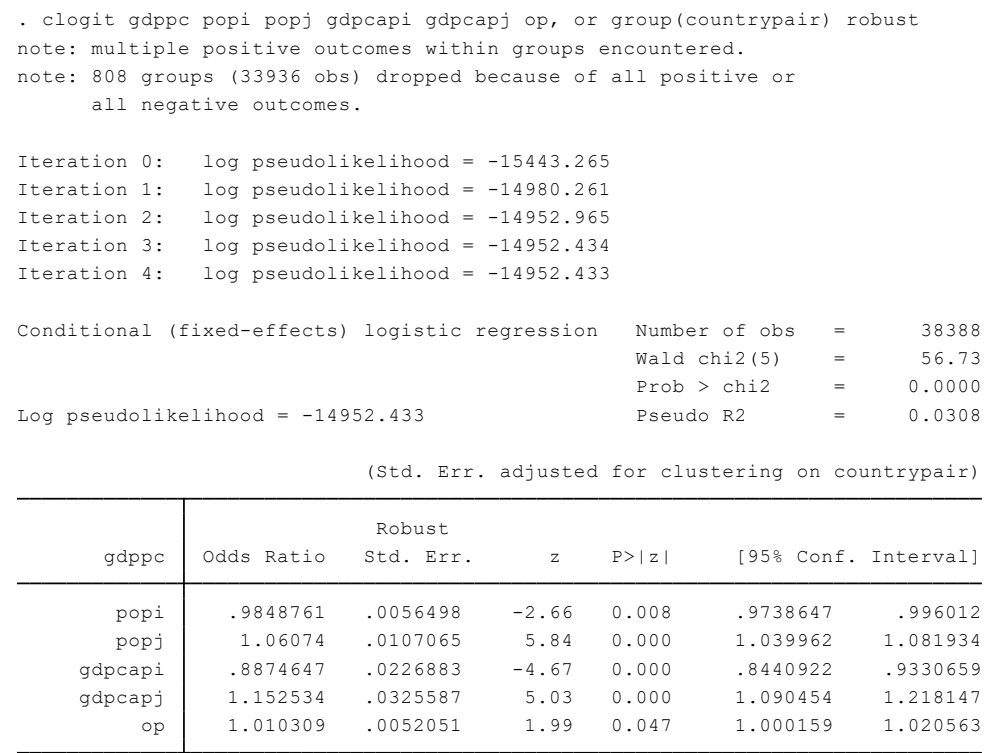

Variables:

gdppc: dependent binary variable, if the value is 1 , the cyclical components of country $i$ granger causes the cyclical components of country $j$, so the shock spreads

gdpcapi: per capita GDP of country $i$

gdpcapj: per capita GDP of country $j$

popi: population in country $i$

popj: population in country $j$

op: the average trade volume between country $i$ and country $j$ divided by the GDP of country $j$

\section{Model - 2A}

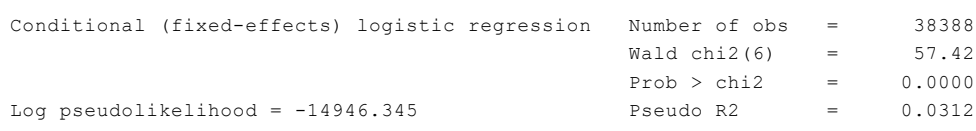

\begin{tabular}{|c|c|c|c|c|c|c|}
\hline gdppc & Odds Ratio & $\begin{array}{l}\text { Robust } \\
\text { Std. Err. }\end{array}$ & $\mathrm{z}$ & $P>|z|$ & [95\% Conf. & Interval] \\
\hline popi & .9852538 & .0056001 & -2.61 & 0.009 & .9743387 & .9962911 \\
\hline popj & 1.060491 & .0106602 & 5.84 & 0.000 & 1.039801 & 1.081592 \\
\hline gdpcapi & .8876793 & .0227075 & -4.66 & 0.000 & .8442706 & .9333198 \\
\hline gdpcapj & 1.15319 & .0327404 & 5.02 & 0.000 & 1.090773 & 1.219179 \\
\hline up & .9990693 & .0071911 & -0.13 & 0.897 & .9850739 & 1.013264 \\
\hline down & 1.010078 & .0086875 & 1.17 & 0.244 & .9931932 & 1.027249 \\
\hline
\end{tabular}

Variables:

up: average trade volume from country $j$ to country $i$ divided by the GDP of country $j$ down: average trade volume from country $i$ to country $j$ divided by the GDP of country $j$ 


\section{Model - 2B}

$\begin{array}{lllr}\text { Conditional (fixed-effects) logistic regression } & \text { Number of obs } & =38388 \\ & \text { Wald chi2 }(5) & = & 55.14 \\ & \text { Prob }>\text { chi2 } & = & 0.0000 \\ \text { Log pseudolikelihood }=-14966.836 & \text { Pseudo R2 } & = & 0.0298\end{array}$

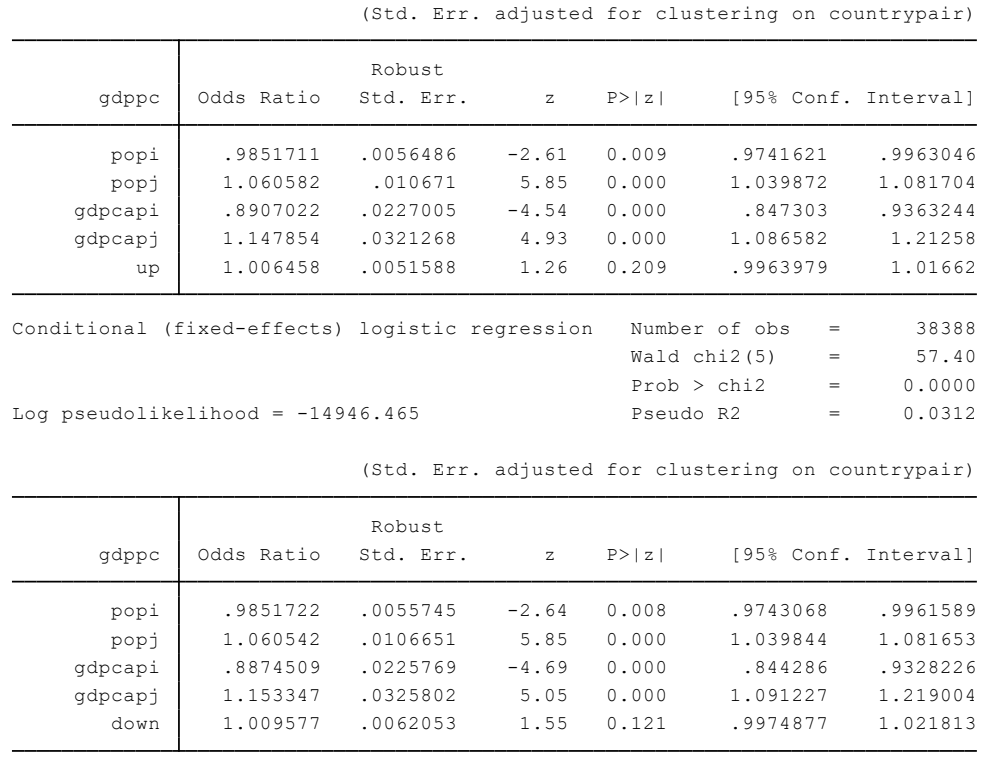

\section{Model - 3A}

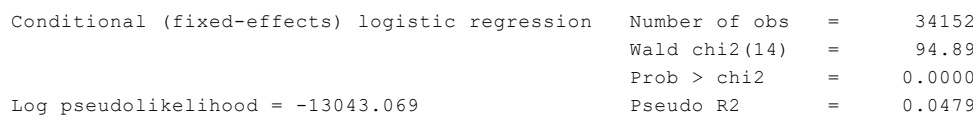

(Std. Err. adjusted for clustering on countrypair)

\begin{tabular}{|c|c|c|c|c|c|c|}
\hline gdppc & Odds Ratio & $\begin{array}{l}\text { Robust } \\
\text { Std. Err. }\end{array}$ & z & $\mathrm{P}>|\mathrm{z}|$ & [95\% Conf. & Interval] \\
\hline popi & .9857961 & .0056572 & -2.49 & 0.013 & .9747703 & .9969466 \\
\hline popj & 1.057231 & .0095421 & 6.17 & 0.000 & 1.038693 & 1.076099 \\
\hline gdpcapi & .8703738 & .02564 & -4.71 & 0.000 & .8215436 & .9221063 \\
\hline gdpcapj & 1.153791 & .0371739 & 4.44 & 0.000 & 1.083184 & 1.229 \\
\hline opo & 1.141174 & .0663777 & 2.27 & 0.023 & 1.018218 & 1.278978 \\
\hline op 1 & .8490814 & .1564053 & -0.89 & 0.374 & .5917707 & 1.218274 \\
\hline$o p 2$ & .8805054 & .0790302 & -1.42 & 0.156 & .7384685 & 1.049862 \\
\hline op3 & .9497904 & .0300455 & -1.63 & 0.103 & .8926907 & 1.010542 \\
\hline op 4 & 2.853801 & 1.385118 & 2.16 & 0.031 & 1.102263 & 7.388595 \\
\hline op 5 & .8841636 & .0356957 & -3.05 & 0.002 & .8168977 & .9569684 \\
\hline op 6 & .9611029 & .0255927 & -1.49 & 0.136 & .9122286 & 1.012596 \\
\hline op 7 & 1.05266 & .0190985 & 2.83 & 0.005 & 1.015886 & 1.090766 \\
\hline op 8 & 1.185209 & .0792218 & 2.54 & 0.011 & 1.039678 & 1.351111 \\
\hline op 9 & .9737451 & .0328815 & -0.79 & 0.431 & .911385 & 1.040372 \\
\hline
\end{tabular}

Variables:

op0: the average trade volume of 'Food and live animals' industry between country $i$ and country $j$ divided by the GDP of country $j$

op1: the average trade volume of 'Beverages and tobacco' industry between country $i$ and country $j$ divided by the GDP of country $j$

op2: the average trade volume of 'Crude materials, inedible, except fuels' industry between country $i$ and country $j$ divided by the GDP of country $j$ 
op3: the average trade volume of 'Mineral fuels, lubricants and related materials' industry between country $i$ and country $j$ divided by the GDP of country $j$

op4: the average trade volume of 'Animal and vegetable oils, fats and waxes' industry between country $i$ and country $j$ divided by the GDP of country $j$

op5: the average trade volume of 'Chemicals and related products, n.e.s.' industry between country $i$ and country $j$ divided by the GDP of country $j$

op6: the average trade volume of 'Manufactured goods classified chiefly by material' industry between country $i$ and country $j$ divided by the GDP of country $j$

op7: the average trade volume of 'Machinery and transport equipment' industry between country $i$ and country $j$ divided by the GDP of country $j$

op8: the average trade volume of 'Miscellaneous manufactured articles' industry between country $i$ and country $j$ divided by the GDP of country $j$

op9: the average trade volume of 'Commodities and transactions not classified elsewhere in the SITC' industry between country $i$ and country $j$ divided by the GDP of country $j$

\section{Model - 3B}

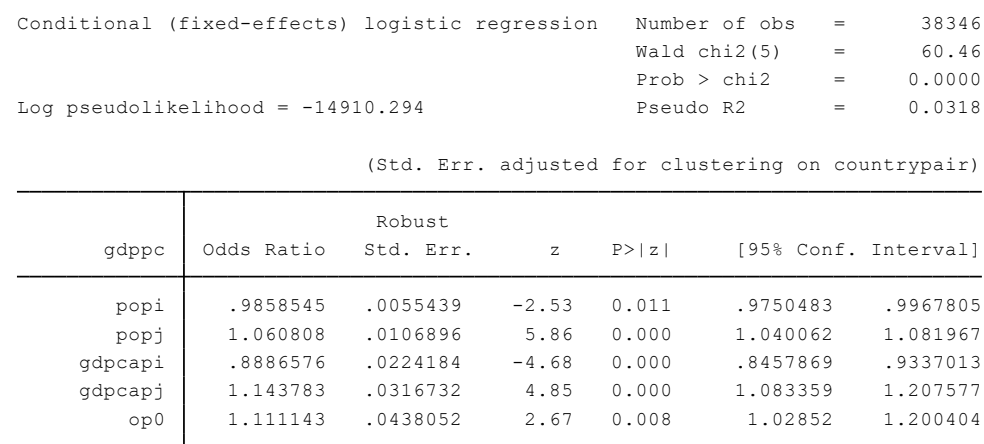

\begin{tabular}{|c|c|c|c|c|c|c|c|c|}
\hline Cond & ditional & ixed-effects) & logistic $r$ & regression & Number & of obs & $=$ & 38041 \\
\hline & & & & & Wald $\mathrm{cl}$ & $\operatorname{li2}(5)$ & $=$ & 56.31 \\
\hline & & & & & Prob > & chi2 & $=$ & 0.0000 \\
\hline Log & pseudolit & lihood $=-148$ & 18.455 & & Pseudo & R2 & $=$ & 0.0296 \\
\hline & & & (Std. Err. & sted & for clust & ering & on $\mathrm{cc}$ & untrypair) \\
\hline & & & Robust & & & & & \\
\hline & gdppc & Odds Ratio & Std. Err. & z & $P>|z|$ & {$[95 \%$} & $\operatorname{Conf}$ & Interval] \\
\hline & popi & .9857111 & .0055721 & -2.55 & 0.011 & .9748 & 8502 & .996693 \\
\hline & popj & 1.058889 & .0101971 & 5.94 & 0.000 & 1.039 & 9091 & 1.079065 \\
\hline & gdpcapi & .8889666 & .0226059 & -4.63 & 0.000 & .8457 & 7459 & .9343961 \\
\hline & gdpcapj & 1.149167 & .0325611 & 4.91 & 0.000 & 1.087 & 7088 & 1.214791 \\
\hline & op 1 & 1.188069 & .1342605 & 1.52 & 0.127 & .9520 & 0276 & 1.482633 \\
\hline
\end{tabular}

$\begin{array}{lll}\text { Conditional (fixed-effects) logistic regression } & \text { Number of obs } & =38346 \\ & \text { Wald chi2 }(5)= & 55.46 \\ & \text { Prob }>\text { chi2 } & =0.0000 \\ \text { Log pseudolikelihood }=-14966.059 & \text { Pseudo } 22 & =0.0293\end{array}$

(Std. Err. adjusted for clustering on countrypair)

\begin{tabular}{|c|c|c|c|c|c|c|}
\hline gdppc & odds Ratio & $\begin{array}{c}\text { Robust } \\
\text { Std. Err. }\end{array}$ & z & $P>|z|$ & [95\% Conf. & Interval] \\
\hline popi & .986206 & .0056021 & -2.45 & 0.014 & .9752869 & .9972473 \\
\hline popj & 1.059989 & .0105344 & 5.86 & 0.000 & 1.039542 & 1.080838 \\
\hline gdpcapi & .8952269 & .022329 & -4.44 & 0.000 & .8525154 & .9400783 \\
\hline gdpcapj & 1.143124 & .0316963 & 4.82 & 0.000 & 1.082658 & 1.206967 \\
\hline op2 & .9705471 & .0785137 & -0.37 & 0.712 & .8282426 & 1.137302 \\
\hline
\end{tabular}




$\begin{array}{llll}\text { Conditional (fixed-effects) logistic regression } & \text { Number of obs } & = & 36610 \\ & \text { Wald chi2 (5) } & = & 57.00 \\ & \text { Prob }>\text { chi2 } & = & 0.0000 \\ \text { Log pseudolikelihood }=-14253.377 & \text { Pseudo R2 } & = & 0.0291\end{array}$

(Std. Err. adjusted for clustering on countrypair)

\begin{tabular}{r|rrrrrr}
\hline & \multicolumn{5}{|c}{ Robust } \\
gdppe & Odds Ratio & Std. Err. & $z$ & P $>|z|$ & [95\% Conf. Interval] \\
\hline popi & .9873803 & .0055938 & -2.24 & 0.025 & .9764772 & .9984051 \\
popj & 1.056118 & .0095515 & 6.04 & 0.000 & 1.037563 & 1.075006 \\
gdpcapi & .8930956 & .0232125 & -4.35 & 0.000 & .8487394 & .93977 \\
gdpcapj & 1.145698 & .0330602 & 4.71 & 0.000 & 1.0827 & 1.212363 \\
op3 & .9821558 & .0291233 & -0.61 & 0.544 & .9267021 & 1.040928 \\
\hline
\end{tabular}

Conditional (fixed-effects) logistic regression Number of obs $=35204$

Wald chi2(5)= 59.40

Prob $>$ chi2 $=0.0000$

Log pseudolikelihood $=-13687.003$

Pseudo R2 $2=0.0327$

(Std. Err. adjusted for clustering on countrypair)

\begin{tabular}{|c|c|c|c|c|c|c|}
\hline \multirow[b]{2}{*}{ gdppc } & \multicolumn{4}{|c|}{ Robust } & \multirow[b]{2}{*}{ [95\% Conf. } & \multirow[b]{2}{*}{ Interval] } \\
\hline & Odds Ratio & Std. Err. & $z$ & $P>|z|$ & & \\
\hline popi & .9830191 & .0056234 & -2.99 & 0.003 & .972059 & .9941028 \\
\hline popj & 1.058782 & .0099109 & 6.10 & 0.000 & 1.039534 & 1.078386 \\
\hline gdpcapi & .8790629 & .0241712 & -4.69 & 0.000 & .8329422 & .9277373 \\
\hline gdpcapj & 1.124352 & .0322865 & 4.08 & 0.000 & 1.062819 & 1.189447 \\
\hline op 4 & 2.902649 & 1.384093 & 2.23 & 0.025 & 1.140012 & 7.390601 \\
\hline
\end{tabular}

Conditional (fixed-effects) logistic regression Number of obs = 38388 Wald $\operatorname{chi2}(5)=60.56$ Prob $>$ chi2 $=0.0000$

Log pseudolikelihood $=-14958.517$

Pseudo R2 $=0.0304$

(Std. Err. adjusted for clustering on countrypair)

\begin{tabular}{|c|c|c|c|c|c|c|c|}
\hline gdppc & Odds Ratio & $\begin{array}{l}\text { Robust } \\
\text { Std. Err. }\end{array}$ & z & $P>|z|$ & {$[95 \%$} & Conf. & Interval] \\
\hline popi & .9864805 & .0055219 & -2.43 & 0.015 & .975 & 5717 & .9973627 \\
\hline popj & 1.059484 & .0104258 & 5.87 & 0.000 & 1.039 & 9245 & 1.080116 \\
\hline gdpcapi & .8983147 & .0225149 & -4.28 & 0.000 & .8552 & 2526 & .943545 \\
\hline gdpcapj & 1.146194 & .032144 & 4.87 & 0.000 & 1.084 & 4893 & 1.210958 \\
\hline op 5 & .9468201 & .0253272 & -2.04 & 0.041 & .8984 & 4585 & .9977849 \\
\hline \multirow[t]{3}{*}{ Conditional } & (fixed-effects) & logistic & regression & Number & of obs & $=$ & 38388 \\
\hline & & & & Wald $\mathrm{ch}$ & $12(5)$ & $=$ & 55.23 \\
\hline & & & & Prob > & chi2 & $=$ & 0.0000 \\
\hline \multicolumn{4}{|c|}{ Log pseudolikelihood $=-14966.424$} & Pseudo & R2 & $=$ & 0.0299 \\
\hline
\end{tabular}

(Std. Err. adjusted for clustering on countrypair)

\begin{tabular}{|c|c|c|c|c|c|c|}
\hline gdppc & odds Ratio & $\begin{array}{l}\text { Robust } \\
\text { Std. Err. }\end{array}$ & z & $P>|z|$ & [95\% Conf. & Interval] \\
\hline popi & .985394 & .0055801 & -2.60 & 0.009 & .9745178 & .9963917 \\
\hline popj & 1.060236 & .0105917 & 5.86 & 0.000 & 1.039679 & 1.0812 \\
\hline gdpcapi & .8911242 & .0226073 & -4.54 & 0.000 & .8478984 & .9365537 \\
\hline gdpcapj & 1.152281 & .0334901 & 4.88 & 0.000 & 1.088476 & 1.219826 \\
\hline op 6 & 1.024584 & .0147582 & 1.69 & 0.092 & .9960628 & 1.053922 \\
\hline
\end{tabular}




$\begin{array}{lllr}\text { Conditional (fixed-effects) logistic regression } & \text { Number of obs } & =38388 \\ & \text { Wald chi2 }(5) & = & 62.28 \\ & \text { Prob }>\text { chi2 } & = & 0.0000 \\ \text { Log pseudolikelihood }=-14900.847 & \text { Pseudo } 2 & = & 0.0341\end{array}$

(Std. Err. adjusted for clustering on countrypair)

\begin{tabular}{|c|c|c|c|c|c|c|}
\hline \multirow[b]{2}{*}{ gdppc } & \multicolumn{3}{|c|}{ Robust } & \multirow[b]{2}{*}{$P>|z|$} & \multirow[b]{2}{*}{$95 \%$} & \multirow[b]{2}{*}{ Interval] } \\
\hline & Odds Ratio & Std. Err. & $z$ & & & \\
\hline popi & .9850459 & .0055629 & -2.67 & 0.008 & .9742031 & .9960095 \\
\hline popj & 1.060974 & .010739 & 5.85 & 0.000 & 1.040133 & 1.082232 \\
\hline gdpcapi & .8827524 & .0225939 & -4.87 & 0.000 & .8395616 & .9281652 \\
\hline gdpcapj & 1.164355 & .0333571 & 5.31 & 0.000 & 1.100778 & 1.231604 \\
\hline op 7 & 1.046708 & .0171913 & 2.78 & 0.005 & 1.01355 & 1.080951 \\
\hline
\end{tabular}

$\begin{array}{llll}\text { Conditional (fixed-effects) logistic regression } & \text { Number of obs } & =38388 \\ & \text { Wald chi2 }(5) & = & 60.97 \\ & \text { Prob }>\text { chi2 } & = & 0.0000 \\ \text { Log pseudolikelihood }=-14917.591 & \text { Pseudo R2 } & = & 0.0330\end{array}$

(Std. Err. adjusted for clustering on countrypair)

\begin{tabular}{|c|c|c|c|c|c|c|c|}
\hline gdppc & odds Ratio & $\begin{array}{l}\text { Robust } \\
\text { Std. Err. }\end{array}$ & $\mathrm{z}$ & $P>|z|$ & {$[95 \%$} & Conf. & Interval] \\
\hline popi & .984942 & .0055664 & -2.68 & 0.007 & .9740 & 0922 & .9959126 \\
\hline popj & 1.060685 & .0106673 & 5.86 & 0.000 & 1.039 & 9982 & 1.0818 \\
\hline gdpcapi & .8853103 & .0227641 & -4.74 & 0.000 & .8417 & 7991 & .9310706 \\
\hline gdpcapj & 1.159347 & .0335502 & 5.11 & 0.000 & 1.09 & 9542 & 1.227004 \\
\hline op 8 & 1.159254 & .051695 & 3.31 & 0.001 & 1.062 & 2235 & 1.265133 \\
\hline \multirow[t]{3}{*}{ Conditional } & (fixed-effects) & logistic & regression & Number & of obs & $=$ & 37961 \\
\hline & & & & wald ch & hi2 (5) & $=$ & 56.72 \\
\hline & & & & Prob > & chi2 & $=$ & 0.0000 \\
\hline \multicolumn{4}{|c|}{ Log pseudolikelihood $=-14805.088$} & Pseudo & R2 & $=$ & 0.0292 \\
\hline
\end{tabular}

(Std. Err. adjusted for clustering on countrypair)

\begin{tabular}{|c|c|c|c|c|c|c|}
\hline gdppc & Odds Ratio & $\begin{array}{l}\text { Robust } \\
\text { Std. Err. }\end{array}$ & z & $P>|z|$ & [95\% Conf. & Interval] \\
\hline popi & .986313 & .0055623 & -2.44 & 0.015 & .9754711 & .9972753 \\
\hline popj & 1.058407 & .0100356 & 5.99 & 0.000 & 1.038919 & 1.07826 \\
\hline gdpcapi & .8942086 & .0223403 & -4.48 & 0.000 & .8514771 & .9390845 \\
\hline gdpcapj & 1.142332 & .0316906 & 4.80 & 0.000 & 1.081878 & 1.206164 \\
\hline op9 & .9784782 & .0272181 & -0.78 & 0.434 & .9265598 & 1.033306 \\
\hline
\end{tabular}




\section{Model - 4A}

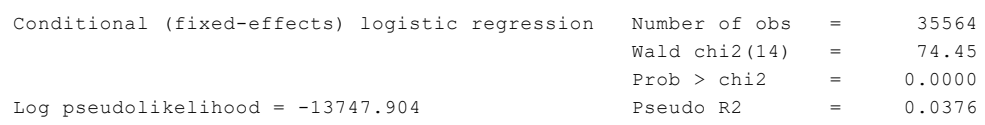

(Std. Err. adjusted for clustering on countrypair)

\begin{tabular}{|c|c|c|c|c|c|c|}
\hline gdppc & Odds Ratio & $\begin{array}{l}\text { Robust } \\
\text { Std. Err. }\end{array}$ & z & $P>|z|$ & [95\% Conf. & Interval] \\
\hline popi & .9856913 & .0056124 & -2.53 & 0.011 & .9747523 & .996753 \\
\hline popj & 1.059549 & .0103332 & 5.93 & 0.000 & 1.039489 & 1.079997 \\
\hline gdpcapi & .8863752 & .0242516 & -4.41 & 0.000 & .8400949 & .9352051 \\
\hline gdpcapj & 1.135106 & .0347337 & 4.14 & 0.000 & 1.06903 & 1.205266 \\
\hline upo & 1.073886 & .0367397 & 2.08 & 0.037 & 1.004238 & 1.148363 \\
\hline up1 & 1.030595 & .1055594 & 0.29 & 0.769 & .8431464 & 1.259717 \\
\hline up2 & .9851659 & .0564616 & -0.26 & 0.794 & .8804922 & 1.102283 \\
\hline up3 & .960096 & .0236402 & -1.65 & 0.098 & .9148623 & 1.007566 \\
\hline up 4 & 1.317129 & .4062586 & 0.89 & 0.372 & .7195838 & 2.410877 \\
\hline up5 & .9512776 & .0215565 & -2.20 & 0.028 & .9099522 & .9944798 \\
\hline up 6 & .9826618 & .0167923 & -1.02 & 0.306 & .9502946 & 1.016131 \\
\hline up7 & 1.035783 & .0153299 & 2.38 & 0.018 & 1.006168 & 1.066269 \\
\hline up 8 & 1.074695 & .036005 & 2.15 & 0.032 & 1.006394 & 1.147632 \\
\hline up 9 & .9820373 & .019604 & -0.91 & 0.364 & .9443562 & 1.021222 \\
\hline
\end{tabular}

Variables:

up0: the average trade volume of 'Food and live animals' industry from country $j$ to country $i$ divided by the GDP of country $j$

up1: the average trade volume of 'Beverages and tobacco' industry from country $j$ to country $i$ divided by the GDP of country $j$

up2: the average trade volume of 'Crude materials, inedible, except fuels' industry from country $j$ to country $i$ divided by the GDP of country $j$

up3: the average trade volume of 'Mineral fuels, lubricants and related materials' industry from country $j$ to country $i$ divided by the GDP of country $j$

up4: the average trade volume of 'Animal and vegetable oils, fats and waxes' industry from country $j$ to country $i$ divided by the GDP of country $j$

up5: the average trade volume of 'Chemicals and related products, $n$.e.s.' industry from country $j$ to country $i$ divided by the GDP of country $j$

up6: the average trade volume of 'Manufactured goods classified chiefly by material' industry from country $j$ to country $i$ divided by the GDP of country $j$

up7: the average trade volume of 'Machinery and transport equipment' industry from country $j$ to country $i$ divided by the GDP of country $j$

up8: the average trade volume of 'Miscellaneous manufactured articles' industry from country $j$ to country $i$ divided by the GDP of country $j$

up9: the average trade volume of 'Commodities and transactions not classified elsewhere in the SITC' industry from country $j$ to country $i$ divided by the GDP of country $j$ 


\begin{tabular}{|c|c|c|c|c|c|c|c|}
\hline \multirow{3}{*}{\multicolumn{2}{|c|}{ ditional }} & \multirow[t]{3}{*}{ fixed-effects) } & \multirow[t]{3}{*}{ logistic $r$} & \multirow[t]{3}{*}{ egression } & \multicolumn{2}{|l|}{ Number } & 5781 \\
\hline & & & & & wald $\mathrm{ch}$ & ni2 (14) & 87.07 \\
\hline & & & & & Prob > & chi2 & 0.0000 \\
\hline \multirow[t]{4}{*}{$\log$} & pseudolik & lihood $=-1373$ & 38.311 & & Pseudo & R2 & 0.0445 \\
\hline & & & (Std. Err. & adjusted & \multicolumn{2}{|l|}{ for } & untrypair) \\
\hline & & & Robust & & & & \\
\hline & gdppc & Odds Ratio & Std. Err. & $z$ & $\mathrm{P}>|\mathrm{z}|$ & [95\% Conf. & Interval] \\
\hline & popi & .9869661 & .0056364 & $-2 \cdot 30$ & 0.022 & .9759805 & .9980753 \\
\hline & popj & 1.05597 & .0093658 & 6.14 & 0.000 & 1.037772 & 1.074487 \\
\hline & gdpcapi & .8722154 & .0244975 & -4.87 & 0.000 & .8254989 & .9215757 \\
\hline & gdpcapj & 1.174567 & .0371524 & 5.09 & 0.000 & 1.103961 & 1.249689 \\
\hline & downo & 1.085316 & .0785232 & 1.13 & 0.258 & .9418269 & 1.250665 \\
\hline & down1 & .7860394 & .1595319 & -1.19 & 0.236 & .528065 & 1.170042 \\
\hline & down2 & .8719528 & .0776846 & -1.54 & 0.124 & .7322462 & 1.038314 \\
\hline & down 3 & .9916915 & .0224786 & -0.37 & 0.713 & .9485986 & 1.036742 \\
\hline & down 4 & 2.525413 & 1.19761 & 1.95 & 0.051 & .9969489 & 6.397232 \\
\hline & down 5 & .8888145 & .0369674 & -2.83 & 0.005 & .8192342 & .9643045 \\
\hline & down 6 & 1.000327 & .0224363 & 0.01 & 0.988 & .9573055 & 1.045282 \\
\hline & down 7 & 1.052102 & .0202159 & 2.64 & 0.008 & 1.013216 & 1.09248 \\
\hline & down8 & 1.056352 & .0689629 & 0.84 & 0.401 & .9294773 & 1.200545 \\
\hline & down 9 & .9964975 & .0155259 & -0.23 & 0.822 & .9665272 & 1.027397 \\
\hline
\end{tabular}

Variables:

down0: the average trade volume of 'Food and live animals' industry from country $i$ to country $j$ divided by the GDP of country $j$

down 1 : the average trade volume of 'Beverages and tobacco' industry from country $i$ to country $j$ divided by the GDP of country $j$

down2: the average trade volume of 'Crude materials, inedible, except fuels' industry from country $i$ to country $j$ divided by the GDP of country $j$

down3: the average trade volume of 'Mineral fuels, lubricants and related materials' industry from country $i$ to country $j$ divided by the GDP of country $j$

down4: the average trade volume of 'Animal and vegetable oils, fats and waxes' industry from country $i$ to country $j$ divided by the GDP of country $j$

down5: the average trade volume of 'Chemicals and related products, n.e.s.' industry from country $i$ to country $j$ divided by the GDP of country $j$

down6: the average trade volume of 'Manufactured goods classified chiefly by material' industry from country $i$ to country $j$ divided by the GDP of country $j$

down7: the average trade volume of 'Machinery and transport equipment' industry from country $i$ to country $j$ divided by the GDP of country $j$

down8: the average trade volume of 'Miscellaneous manufactured articles' industry from country $i$ to country $j$ divided by the GDP of country $j$

down9: the average trade volume of 'Commodities and transactions not classified elsewhere in the SITC' industry from country $i$ to country $j$ divided by the GDP of country $j$ 


\section{Model - 4B}

$\begin{array}{lll}\text { Conditional (fixed-effects) logistic regression } & \text { Number of obs } & =38388 \\ & \text { Wald chi2 }(5)= & 59.69 \\ & \text { Prob }>\text { chi2 } & =0.0000 \\ \text { Log pseudolikelihood }=-14941.317 & \text { Pseudo } 22 & =0.0315\end{array}$

(Std. Err. adjusted for clustering on countrypair)

\begin{tabular}{|c|c|c|c|c|c|c|}
\hline gdpoc & Odds Ratio & Robust & $z$ & $P>|z|$ & {$[95 \%$ Conf } & Interval1 \\
\hline popi & .9859078 & .0055283 & -2.53 & 0.011 & .9751319 & .9968028 \\
\hline popj & 1.060567 & .0106608 & 5.85 & 0.000 & 1.039876 & 1.081668 \\
\hline gdpcapi & .8917215 & .0223666 & -4.57 & 0.000 & .8489439 & .9366547 \\
\hline gdpcapj & 1.143838 & .0316455 & 4.86 & 0.000 & 1.083465 & 1.207574 \\
\hline upo & 1.073003 & .0318894 & 2.37 & 0.018 & 1.012287 & 1.137361 \\
\hline
\end{tabular}

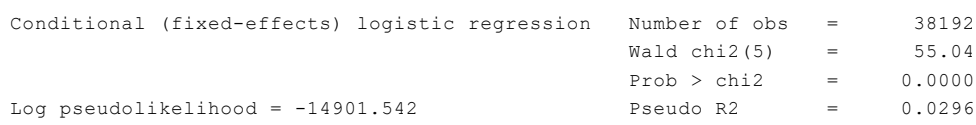

(Std. Err. adjusted for clustering on countrypair)

\begin{tabular}{|c|c|c|c|c|c|c|}
\hline gdppc & Odds Ratio & $\begin{array}{l}\text { Robust } \\
\text { Std. Err. }\end{array}$ & z & $P>|z|$ & [95\% Conf. & Interval] \\
\hline popi & .9858536 & .005551 & -2.53 & 0.011 & .9750337 & .9967937 \\
\hline popj & 1.060305 & .0106086 & 5.85 & 0.000 & 1.039715 & 1.081302 \\
\hline gdpcapi & .8934555 & .0223987 & -4.49 & 0.000 & .8506159 & .9384526 \\
\hline gdpcapj & 1.144344 & .031732 & 4.86 & 0.000 & 1.08381 & 1.208259 \\
\hline up1 & 1.06526 & .0930463 & 0.72 & 0.469 & .8976484 & 1.264167 \\
\hline
\end{tabular}

Conditional (fixed-effects) logistic regression

Tald chi2 $(5)=55.10$

Prob $>$ chi2 $=0.0000$

Log pseudolikelihood $=-14975.988$

Pseudo R2 $=0.0293$

(Std. Err. adjusted for clustering on countrypair)

\begin{tabular}{|c|c|c|c|c|c|c|}
\hline gdppc & Odds Ratio & $\begin{array}{l}\text { Robust } \\
\text { Std. Err. }\end{array}$ & z & $P>|z|$ & [95\% Conf. & Interval] \\
\hline popi & .9859001 & .0056409 & -2.48 & 0.013 & .974906 & .9970182 \\
\hline popj & 1.060067 & .0105538 & 5.86 & 0.000 & 1.039582 & 1.080955 \\
\hline gdpcapi & .8946512 & .0223088 & -4.46 & 0.000 & .851978 & .9394616 \\
\hline gdpcapj & 1.143789 & .0316891 & 4.85 & 0.000 & 1.083336 & 1.207616 \\
\hline up2 & 1.008123 & .0548569 & 0.15 & 0.882 & .9061408 & 1.121584 \\
\hline
\end{tabular}

Conditional (fixed-effects) logistic regression Number of obs $=$ Wald $\operatorname{chi}(5)=57.12$ Prob > chi2 $=0.0000$

Log pseudolikelihood $=-14570.644$

Pseudo R2 $=$

(Std. Err. adjusted for clustering on countrypair)

\begin{tabular}{|c|c|c|c|c|c|c|c|}
\hline gdppc & Odds Ratio & $\begin{array}{l}\text { Robust } \\
\text { Std. Err. }\end{array}$ & $\mathrm{z}$ & $P>|z|$ & {$[95 \%$} & Conf. & Interval] \\
\hline popi & .9865566 & .0055377 & -2.41 & 0.016 & .9757 & 7623 & .9974703 \\
\hline popj & 1.057754 & .0100361 & 5.92 & 0.000 & 1.038 & 8266 & 1.077609 \\
\hline gdpcapi & .8936903 & .0229082 & -4.38 & 0.000 & .8490 & 9004 & .9397365 \\
\hline gdpcapj & 1.144003 & .0319664 & 4.81 & 0.000 & 1.083 & 3035 & 1.208404 \\
\hline up3 & .9725303 & .0257061 & -1.05 & 0.292 & .9234 & 4302 & 1.024241 \\
\hline
\end{tabular}




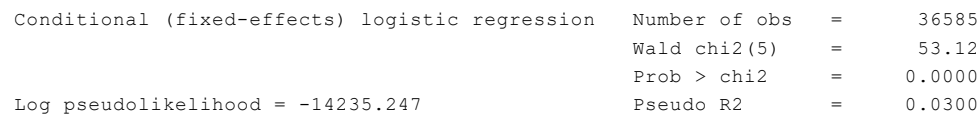

(Std. Err. adjusted for clustering on countrypair)

\begin{tabular}{r|rrrrrr}
\hline & \multicolumn{5}{|c}{ Robust } \\
gdppe & Odds Ratio & Std. Err. & $z$ & P $>|z|$ & [95\% Conf. Interval] \\
\hline popi & .9835259 & .0055841 & -2.93 & 0.003 & .9726419 & .9945316 \\
popj & 1.060954 & .0106469 & 5.90 & 0.000 & 1.04029 & 1.082028 \\
gdpcapi & .8945889 & .022936 & -4.34 & 0.000 & .8507459 & .9406913 \\
gdpcapj & 1.121703 & .0317395 & 4.06 & 0.000 & 1.061188 & 1.185668 \\
up4 & 1.521745 & .4572674 & 1.40 & 0.162 & .844433 & 2.742322 \\
\hline
\end{tabular}

$\begin{array}{lllr}\text { Conditional (fixed-effects) logistic regression } & \text { Number of obs } & =38388 \\ & \text { Wald chi2 }(5) & = & 58.05 \\ & \text { Prob }>\text { chi2 } & = & 0.0000 \\ \text { Log pseudolikelihood }=-14965.221 & \text { Pseudo R2 } & = & 0.0300\end{array}$

(Std. Err. adjusted for clustering on countrypair)

\begin{tabular}{|c|c|c|c|c|c|c|c|}
\hline gdppc & Odds Ratio & $\begin{array}{l}\text { Robust } \\
\text { Std. Err. }\end{array}$ & z & $P>|z|$ & {$[95 \%$} & Conf. & Interval] \\
\hline popi & .9861932 & .005517 & -2.49 & 0.013 & .9754 & 4392 & .9970658 \\
\hline popj & 1.059736 & .0104736 & 5.87 & 0.000 & 1.039 & 9406 & 1.080464 \\
\hline gdpcapi & .8964258 & .0223799 & -4.38 & 0.000 & .8536 & 6179 & .9413804 \\
\hline gdpcapj & 1.14543 & .0321147 & 4.84 & 0.000 & 1.084 & 4184 & 1.210135 \\
\hline up5 & .9695977 & .0160408 & -1.87 & 0.062 & .9386 & 6625 & 1.001552 \\
\hline \multirow[t]{3}{*}{ Conditional } & (fixed-effects) & logistic & regression & Number & of obs & $=$ & 38388 \\
\hline & & & & Wald $\mathrm{ch}$ & $12(5)$ & $=$ & 55.15 \\
\hline & & & & Prob > & chi2 & $=$ & 0.0000 \\
\hline \multicolumn{4}{|c|}{ Log pseudolikelihood $=-14974.245$} & Pseudo & R2 & $=$ & 0.0294 \\
\hline
\end{tabular}

(Std. Err. adjusted for clustering on countrypair)

\begin{tabular}{|c|c|c|c|c|c|c|}
\hline & & Robust & & & & \\
\hline gdppc & odds Ratio & Std. Err. & $\mathrm{z}$ & $P>|z|$ & {$[95 \%$ Conf } & Interval] \\
\hline popi & .9857588 & .0055662 & -2.54 & 0.011 & .9749093 & .9967289 \\
\hline popj & 1.060166 & .0105762 & 5.86 & 0.000 & 1.039638 & 1.081099 \\
\hline gdpcapi & .8932035 & .0225838 & -4.47 & 0.000 & .8500189 & .938582 \\
\hline gdpcapj & 1.147196 & .0330537 & 4.77 & 0.000 & 1.084207 & 1.213844 \\
\hline up 6 & 1.008651 & .0116459 & 0.75 & 0.456 & .9860815 & 1.031736 \\
\hline
\end{tabular}

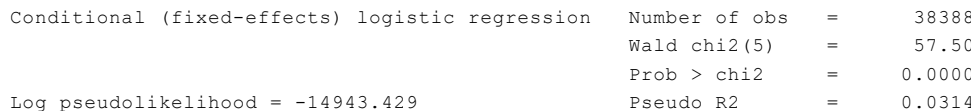

(Std. Err. adjusted for clustering on countrypair)

\begin{tabular}{|c|c|c|c|c|c|c|}
\hline gdppc & Odds Ratio & $\begin{array}{c}\text { Robust } \\
\text { Std. Err. }\end{array}$ & z & $\mathrm{P}>|\mathrm{z}|$ & [95\% Conf. & Interval] \\
\hline popi & .9855255 & .0055506 & -2.59 & 0.010 & .9747064 & .9964647 \\
\hline popj & 1.06088 & .010731 & 5.84 & 0.000 & 1.040055 & 1.082122 \\
\hline gdpcapi & .8882761 & .0224461 & -4.69 & 0.000 & .8453542 & .9333772 \\
\hline gdpcapj & 1.151536 & .0318629 & 5.10 & 0.000 & 1.090749 & 1.21571 \\
\hline up7 & 1.028403 & .0147894 & 1.95 & 0.051 & .9998211 & 1.057802 \\
\hline
\end{tabular}




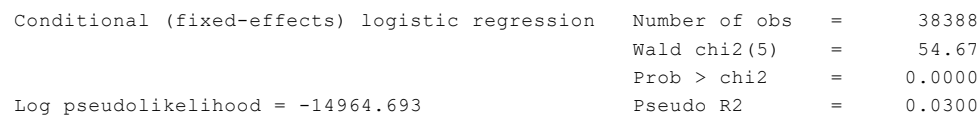

(Std. Err. adjusted for clustering on countrypair)

\begin{tabular}{|c|c|c|c|c|c|c|}
\hline \multirow[b]{2}{*}{ gdppc } & \multicolumn{3}{|c|}{ Robust } & \multirow[b]{2}{*}{$\mathrm{P}>|\mathrm{z}|$} & \multirow[b]{2}{*}{$95 \%$} & \multirow[b]{2}{*}{ Interval] } \\
\hline & Odds Ratio & Std. Err. & $z$ & & & \\
\hline popi & .9857523 & .0055494 & -2.55 & 0.011 & .9749355 & .9966892 \\
\hline popj & 1.060213 & .0105814 & 5.86 & 0.000 & 1.039676 & 1.081157 \\
\hline gdpcapi & .8926549 & .0226592 & -4.47 & 0.000 & .8493304 & .9381894 \\
\hline gdpcapj & 1.146958 & .0328369 & 4.79 & 0.000 & 1.084371 & 1.213157 \\
\hline up8 & 1.04944 & .0451683 & 1.12 & 0.262 & .9645426 & 1.141809 \\
\hline
\end{tabular}

$\begin{array}{lllr}\text { Conditional (fixed-effects) logistic regression } & \text { Number of obs } & = & 38143 \\ & \text { Wald chi2 (5) } & = & 55.24 \\ & \text { Prob }>\text { chi2 } & = & 0.0000 \\ \text { Log pseudolikelihood }=-14867.108 & \text { Pseudo R2 } & = & 0.0295\end{array}$

(Std. Err. adjusted for clustering on countrypair)

\begin{tabular}{r|rrrrrr}
\hline & \multicolumn{5}{|c}{ Robust } \\
gdppe & Odds Ratio & Std. Err. & $z$ & P >|z| & [95\% Conf. Interval] \\
\hline popi & .9863653 & .0055791 & -2.43 & 0.015 & .9754908 & .997361 \\
popj & 1.060127 & .010559 & 5.86 & 0.000 & 1.039633 & 1.081026 \\
gdpcapi & .8949431 & .0223176 & -4.45 & 0.000 & .8522531 & .9397715 \\
gdpcapj & 1.140714 & .0314709 & 4.77 & 0.000 & 1.08067 & 1.204093 \\
up9 & .9865241 & .0170939 & -0.78 & 0.434 & .9535832 & 1.020603 \\
\hline
\end{tabular}

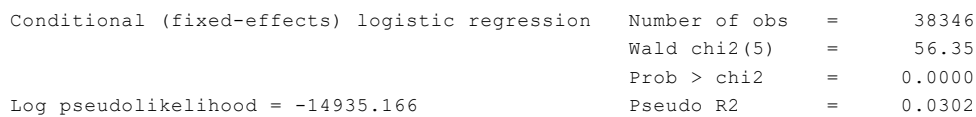

(Std. Err. adjusted for clustering on countrypair)

\begin{tabular}{r|rrrrrr}
\hline \multirow{2}{*}{ gdppc } & Odds Ratio & $\begin{array}{c}\text { Robust } \\
\text { Std. Err. }\end{array}$ & $z$ & P $>|z|$ & [95\% Conf. Interval] \\
\hline popi & .9859122 & .0055461 & -2.52 & 0.012 & .9751018 & .9968425 \\
popj & 1.060532 & .0106301 & 5.86 & 0.000 & 1.039901 & 1.081572 \\
gdpcapi & .8904293 & .0224109 & -4.61 & 0.000 & .8475705 & .9354553 \\
gdpcapj & 1.143301 & .0316297 & 4.84 & 0.000 & 1.082959 & 1.207006 \\
down0 & 1.063278 & .0433756 & 1.50 & 0.133 & .9815734 & 1.151784 \\
\hline
\end{tabular}

Conditional (fixed-effects) logistic regression Number of obs $=$ Wald $\operatorname{chi} 2(5)=56.38$ Prob $>$ chi2 $=0.0000$ Log pseudolikelihood $=-14890.777 \quad$ Pseudo $R 2=0.0294$

(Std. Err. adjusted for clustering on countrypair)

\begin{tabular}{|c|c|c|c|c|c|c|}
\hline \multirow[b]{2}{*}{ gdppc } & \multicolumn{4}{|c|}{ Robust } & \multirow[b]{2}{*}{$95 \%$} & \multirow[b]{2}{*}{ Interval] } \\
\hline & Odds Ratio & Std. Err. & z & $\mathrm{P}>|\mathrm{z}|$ & & \\
\hline popi & .985791 & .0055556 & -2.54 & 0.011 & .9749621 & .9967401 \\
\hline popj & 1.058687 & .0101475 & 5.95 & 0.000 & 1.038983 & 1.078763 \\
\hline gdpcapi & .8892639 & .022586 & -4.62 & 0.000 & .8460798 & .934652 \\
\hline gdpcapj & 1.150382 & .0329173 & 4.90 & 0.000 & 1.087641 & 1.216743 \\
\hline down 1 & 1.14929 & .1102872 & 1.45 & 0.147 & .9522417 & 1.387113 \\
\hline
\end{tabular}




$\begin{array}{lllr}\text { Conditional (fixed-effects) logistic regression } & \text { Number of obs } & =38346 \\ & \text { Wald chi2 }(5) & = & 55.66 \\ & \text { Prob }>\text { chi2 } & = & 0.0000 \\ \text { Log pseudolikelihood }=-14963.18 & \text { Pseudo R2 } & = & 0.0295\end{array}$

(Std. Err. adjusted for clustering on countrypair)

\begin{tabular}{|c|c|c|c|c|c|c|}
\hline gdppc & Odds Ratio & $\begin{array}{l}\text { Robust } \\
\text { Std. Err. }\end{array}$ & z & $P>|z|$ & [95\% Conf. & Interval] \\
\hline popi & .9860955 & .0055325 & -2.50 & 0.013 & .9753114 & .9969988 \\
\hline popj & 1.059905 & .0105042 & 5.87 & 0.000 & 1.039516 & 1.080694 \\
\hline gdpcapi & .8959104 & .022298 & -4.42 & 0.000 & .853256 & .940697 \\
\hline gdpcapj & 1.142803 & .031729 & 4.81 & 0.000 & 1.082277 & 1.206714 \\
\hline down2 & .9489004 & .0612632 & -0.81 & 0.417 & .8361133 & 1.076902 \\
\hline
\end{tabular}

Conditional (fixed-effects) logistic regression Number of obs $=37502$

Wald chi2(5) $=55.99$

Prob $>$ chi2 $=0.0000$

Log pseudolikelihood $=-14598.756$

Pseudo R2 $2=0.0289$

(Std. Err. adjusted for clustering on countrypair)

\begin{tabular}{|c|c|c|c|c|c|c|}
\hline \multirow[b]{2}{*}{ gdppc } & \multicolumn{4}{|c|}{ Robust } & \multirow[b]{2}{*}{$95 \%$} & \multirow[b]{2}{*}{ Interval] } \\
\hline & Odds Ratio & Std. Err. & $z$ & $P>|z|$ & & \\
\hline popi & .9869702 & .0055979 & -2.31 & 0.021 & .9760592 & .9980032 \\
\hline popj & 1.057503 & .0098685 & 5.99 & 0.000 & 1.038337 & 1.077023 \\
\hline gdpcapi & .894909 & .0226148 & -4.39 & 0.000 & .8516645 & .9403493 \\
\hline gdpcapj & 1.145155 & .0327264 & 4.74 & 0.000 & 1.082776 & 1.211128 \\
\hline down 3 & .9963285 & .0176117 & -0.21 & 0.835 & .9624012 & 1.031452 \\
\hline
\end{tabular}

Conditional (fixed-effects) logistic regression Number of obs $=\quad 36555$ Wald $\operatorname{chi2}(5)=61.37$ Prob $>$ chi2 $=0.0000$

Log pseudolikelihood $=-14264.069$

Pseudo R2 $=0.0327$

(Std. Err. adjusted for clustering on countrypair)

\begin{tabular}{r|rrrrrr}
\hline \multirow{2}{*}{ gdppc } & Odds Ratio & Robust & & & \\
& Std. Err. & $z$ & P $>|z|$ & [95\% Conf. Interval] \\
\hline popi & .985764 & .0055802 & -2.53 & 0.011 & .9748873 & .9967619 \\
popj & 1.057747 & .009804 & 6.06 & 0.000 & 1.038705 & 1.077138 \\
gdpcapi & .8778869 & .0235494 & -4.86 & 0.000 & .8329233 & .9252777 \\
gdpcapj & 1.143062 & .0322054 & 4.75 & 0.000 & 1.081651 & 1.207958 \\
down4 & 2.541145 & 1.26066 & 1.88 & 0.060 & .9610571 & 6.719078 \\
\hline
\end{tabular}

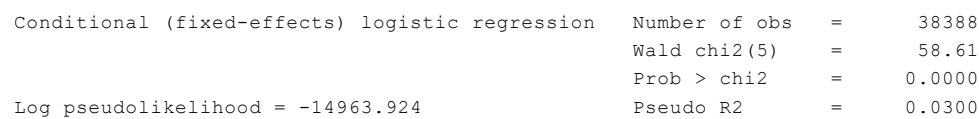

(Std. Err. adjusted for clustering on countrypair)

\begin{tabular}{|c|c|c|c|c|c|c|}
\hline gdppc & Odds Ratio & $\begin{array}{l}\text { Robust } \\
\text { Std. Err. }\end{array}$ & z & $P>|z|$ & [95\% Conf. & Interval] \\
\hline popi & .9864712 & .0055359 & -2.43 & 0.015 & .9756805 & .9973814 \\
\hline popj & 1.059595 & .0104611 & 5.86 & 0.000 & 1.039289 & 1.080299 \\
\hline gdpcapi & .8979779 & .0225414 & -4.29 & 0.000 & .8548668 & .9432632 \\
\hline gdpcapj & 1.145393 & .0318414 & 4.88 & 0.000 & 1.084655 & 1.209532 \\
\hline down 5 & .9578697 & .029274 & -1.41 & 0.159 & .9021782 & 1.016999 \\
\hline
\end{tabular}




$\begin{array}{llll}\text { Conditional (fixed-effects) logistic regression } & \text { Number of obs } & = & 38388 \\ & \text { Wald chi2 (5) } & = & 56.28 \\ & \text { Prob }>\text { chi2 } & = & 0.0000 \\ \text { Log pseudolikelihood }=-14953.633 & \text { Pseudo } 2 & = & 0.0307\end{array}$

(Std. Err. adjusted for clustering on countrypair)

\begin{tabular}{|c|c|c|c|c|c|c|}
\hline \multirow[b]{2}{*}{ gdppc } & \multicolumn{3}{|c|}{ Robust } & \multirow[b]{2}{*}{$\mathrm{P}>|\mathrm{z}|$} & \multirow[b]{2}{*}{$95 \%$} & \multirow[b]{2}{*}{ Interval] } \\
\hline & Odds Ratio & Std. Err. & $z$ & & & \\
\hline popi & .9851522 & .0055659 & -2.65 & 0.008 & .9743034 & .9961218 \\
\hline popj & 1.060115 & .0105653 & 5.86 & 0.000 & 1.039608 & 1.081026 \\
\hline gdpcapi & .8903874 & .0226045 & -4.57 & 0.000 & .8471675 & .9358122 \\
\hline gdpcapj & 1.154903 & .033531 & 4.96 & 0.000 & 1.091018 & 1.222528 \\
\hline down 6 & 1.040464 & .0230842 & 1.79 & 0.074 & .9961896 & 1.086706 \\
\hline
\end{tabular}

$\begin{array}{llll}\text { Conditional (fixed-effects) logistic regression } & \text { Number of obs } & =38388 \\ & \text { Wald chi2 }(5) & = & 62.20 \\ & \text { Prob }>\text { chi2 } & = & 0.0000 \\ \text { Log pseudolikelihood }=-14885.912 & \text { Pseudo R2 } & = & 0.0351\end{array}$

(Std. Err. adjusted for clustering on countrypair)

\begin{tabular}{|c|c|c|c|c|c|c|c|}
\hline gdppc & Odds Ratio & $\begin{array}{l}\text { Robust } \\
\text { Std. Err. }\end{array}$ & $\mathrm{z}$ & $P>|z|$ & {$[95 \%$} & Conf. & Interval] \\
\hline popi & .9850042 & .0055561 & -2.68 & 0.007 & .9741 & 1744 & .9959545 \\
\hline popj & 1.06053 & .0106333 & 5.86 & 0.000 & 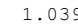 & 9893 & 1.081577 \\
\hline gdpcapi & .8826803 & .0225828 & -4.88 & 0.000 & 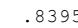 & 5103 & .9280701 \\
\hline gdpcapj & 1.168846 & .0338437 & 5.39 & 0.000 & $1.104 \mathrm{rat} \mathrm{rat}$ & 4361 & 1.237097 \\
\hline down 7 & 1.044657 & .0207124 & 2.20 & 0.028 & 1.00 & 0484 & 1.086052 \\
\hline \multirow[t]{3}{*}{ Conditional } & (fixed-effects) & logistic & regression & Number & of obs & $=$ & 38388 \\
\hline & & & & Wald $\mathrm{ch}$ & hi2 (5) & $=$ & 60.20 \\
\hline & & & & Prob > & chi2 & $=$ & 0.0000 \\
\hline \multicolumn{4}{|c|}{ Log pseudolikelihood $=-14920.833$} & Pseudo & R2 & $=$ & 0.0328 \\
\hline
\end{tabular}

(Std. Err. adjusted for clustering on countrypair)

\begin{tabular}{|c|c|c|c|c|c|c|}
\hline gdppc & odds Ratio & $\begin{array}{l}\text { Robust } \\
\text { Std. Err. }\end{array}$ & z & $P>|z|$ & [95\% Conf. & Interval] \\
\hline popi & .9850689 & .0055382 & -2.68 & 0.007 & .9742738 & .9959835 \\
\hline popj & 1.060563 & .0106404 & 5.86 & 0.000 & 1.039912 & 1.081624 \\
\hline gdpcapi & .8860625 & .0225726 & -4.75 & 0.000 & .8429074 & .9314272 \\
\hline gdpcapj & 1.158869 & .0331514 & 5.15 & 0.000 & 1.095682 & 1.225701 \\
\hline down 8 & 1.10581 & .0584893 & 1.90 & 0.057 & .9969147 & 1.226599 \\
\hline
\end{tabular}

Conditional (fixed-effects) logistic regression Number of obs $=38125$

Wald chi2(5) =
Prob $>$ chi2 $=0.0000$

Log pseudolikelihood $=-14881.213$

Pseudo R2 $=0.0291$

(Std. Err. adjusted for clustering on countrypair)

\begin{tabular}{|c|c|c|c|c|c|c|}
\hline gdppc & Odds Ratio & $\begin{array}{l}\text { Robust } \\
\text { Std. Err. }\end{array}$ & $\mathrm{z}$ & $P>|z|$ & [95\% Conf. & Interval] \\
\hline popi & .9860462 & .0055251 & -2.51 & 0.012 & .9752764 & .9969349 \\
\hline popj & 1.058399 & .0100289 & 5.99 & 0.000 & 1.038924 & 1.078239 \\
\hline gdpcapi & .8929571 & .0223042 & -4.53 & 0.000 & .8502945 & .9377602 \\
\hline gdpcapj & 1.14542 & .0318349 & 4.89 & 0.000 & 1.084693 & 1.209546 \\
\hline down 9 & .9894015 & .02401 & -0.44 & 0.661 & .9434443 & 1.037597 \\
\hline
\end{tabular}




\section{Model - 5A}

\begin{tabular}{|c|c|c|c|c|c|}
\hline \multirow[t]{3}{*}{ Conditional } & (fixed-effects) & logistic regression & Number of obs & $=$ & 34152 \\
\hline & & & Wald chi2 (24) & $=$ & 103.68 \\
\hline & & & Prob > chi2 & $=$ & 0.0000 \\
\hline Log pseudoli & kelihood $=-13$ & 03.79 & Pseudo R2 & $=$ & 0.0508 \\
\hline
\end{tabular}

(Std. Err. adjusted for clustering on countrypair)

\begin{tabular}{|c|c|c|c|c|c|c|}
\hline \multirow[b]{2}{*}{ gdppe } & \multicolumn{3}{|c|}{ Robust } & \multirow[b]{2}{*}{$\mathrm{P}>|\mathrm{z}|$} & \multirow[b]{2}{*}{ [95\% Conf. } & \multirow[b]{2}{*}{ Interval] } \\
\hline & Odds Ratio & Std. Err. & $z$ & & & \\
\hline popi & .9862904 & .0057434 & -2.37 & 0.018 & .9750975 & .9976118 \\
\hline popj & 1.057007 & .0095861 & 6.11 & 0.000 & 1.038384 & 1.075963 \\
\hline gdpcapi & .8716151 & .0258194 & -4.64 & 0.000 & .822451 & .9237181 \\
\hline gdpcapj & 1.153894 & .0381257 & 4.33 & 0.000 & 1.081537 & 1.231092 \\
\hline down 0 & 1.115541 & .0801347 & 1.52 & 0.128 & .9690356 & 1.284197 \\
\hline down 1 & .7752998 & .1380634 & -1.43 & 0.153 & .5468773 & 1.099131 \\
\hline down2 & .8568466 & .0696757 & -1.90 & 0.057 & .7306113 & 1.004893 \\
\hline down 3 & .987575 & .0238001 & -0.52 & 0.604 & .9420123 & 1.035342 \\
\hline down 4 & 2.66333 & 1.301785 & 2.00 & 0.045 & 1.021819 & 6.941862 \\
\hline down 5 & .9010023 & .03897 & -2.41 & 0.016 & .8277704 & .9807129 \\
\hline down 6 & .9897329 & .0297168 & -0.34 & 0.731 & .9331697 & 1.049725 \\
\hline down 7 & 1.046509 & .0228644 & 2.08 & 0.037 & 1.002641 & 1.092296 \\
\hline down 8 & 1.051929 & .0623129 & 0.85 & 0.393 & .936621 & 1.181432 \\
\hline down9 & 1.001987 & .0132906 & 0.15 & 0.881 & .9762739 & 1.028378 \\
\hline upo & 1.069324 & .0356193 & 2.01 & 0.044 & 1.001742 & 1.141466 \\
\hline up1 & 1.012197 & .1159974 & 0.11 & 0.916 & .8085702 & 1.267105 \\
\hline up2 & .983808 & .0532663 & -0.30 & 0.763 & .8847565 & 1.093949 \\
\hline up3 & .9500155 & .0242293 & -2.01 & 0.044 & .9036943 & .9987111 \\
\hline up 4 & 1.353834 & .4159094 & 0.99 & 0.324 & .741428 & 2.472076 \\
\hline up5 & .9663035 & .0214029 & -1.55 & 0.122 & .925252 & 1.009176 \\
\hline up6 & .9829626 & .026643 & -0.63 & 0.526 & .932106 & 1.036594 \\
\hline up7 & 1.014763 & .0142438 & 1.04 & 0.296 & .9872259 & 1.043067 \\
\hline up 8 & 1.096142 & .0480623 & 2.09 & 0.036 & 1.005876 & 1.194508 \\
\hline up9 & .98262 & .0197779 & -0.87 & 0.384 & .9446107 & 1.022159 \\
\hline
\end{tabular}

\section{Model - 5B}

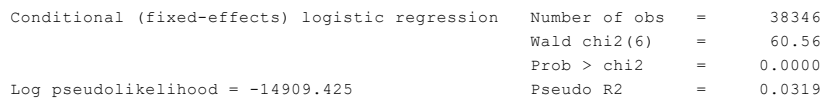

\begin{tabular}{|c|c|c|c|c|c|c|}
\hline gdppc & Odds Ratio & $\begin{array}{l}\text { Robust } \\
\text { Std. Err. }\end{array}$ & z & $P>|z|$ & [95\% Conf. & Interval] \\
\hline popi & 9858709 & .0055403 & -2.53 & 0.011 & .9750717 & .9967896 \\
\hline popj & 1.060755 & .0106786 & 5.86 & 0.000 & 1.04003 & 1.081892 \\
\hline gdpcapi & .8890007 & .0224794 & -4.65 & 0.000 & .846016 & .9341695 \\
\hline gdpcapj & 1.14387 & .031599 & 4.87 & 0.000 & 1.083584 & 1.20751 \\
\hline up 0 & 1.063658 & .0308427 & 2.13 & 0.033 & 1.004893 & 1.12586 \\
\hline down 0 & 1.038682 & .0419437 & 0.94 & 0.347 & .9596426 & 1.12423 \\
\hline
\end{tabular}

$\begin{array}{llll}\text { Conditional (fixed-effects) logistic regression } & \text { Number of obs } & =38041 \\ & \text { Wald chi2 }(6) & =56.37 \\ & \text { Prob }>\text { chi2 } & = & 0.0000 \\ \text { Log pseudolikelihood }=-14816.998 & \text { Pseudo R2 } & =0.0297\end{array}$

\begin{tabular}{r|rrrrrr}
\multicolumn{7}{c}{ (Std. Err. adjusted for clustering on countrypair) } \\
\hline \multirow{2}{*}{ gdppc } & Odds Ratio & $\begin{array}{c}\text { Robust } \\
\text { Std. Err. }\end{array}$ & $z$ & P>|z| & [95\% Conf. Interval] \\
\hline popi & .9856982 & .0055737 & -2.55 & 0.011 & .9748342 & .9966832 \\
popj & 1.058883 & .0101931 & 5.94 & 0.000 & 1.039092 & 1.079051 \\
gdpcapi & .888271 & .0226634 & -4.64 & 0.000 & .8449439 & .9338198 \\
gdpcapj & 1.150865 & .0330227 & 4.90 & 0.000 & 1.087928 & 1.217442 \\
up1 & 1.033896 & .0978178 & 0.35 & 0.725 & .8589029 & 1.244543 \\
down1 & 1.142897 & .1137813 & 1.34 & 0.180 & .9402978 & 1.389148 \\
\hline
\end{tabular}




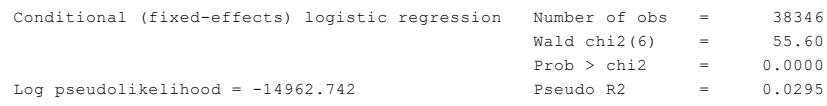

(Std. Err. adjusted for clustering on countrypair)

\begin{tabular}{|c|c|c|c|c|c|c|}
\hline gdppc & Odds Ratio & $\begin{array}{l}\text { Robust } \\
\text { Std. Err. }\end{array}$ & z & $P>|z|$ & [95\% Conf. & Interval] \\
\hline popi & .985919 & .0056483 & -2.48 & 0.013 & .9749105 & .9970519 \\
\hline popj & 1.059928 & .010508 & 5.87 & 0.000 & 1.039532 & 1.080725 \\
\hline gdpcapi & .8957262 & .0223234 & -4.42 & 0.000 & .8530245 & .9405655 \\
\hline gdpcapj & 1.142967 & .0317759 & 4.81 & 0.000 & 1.082354 & 1.206974 \\
\hline up2 & 1.015512 & .0544181 & 0.29 & 0.774 & .9142644 & 1.127972 \\
\hline down 2 & .9461335 & .0606599 & -0.86 & 0.388 & .834409 & 1.072818 \\
\hline
\end{tabular}

Conditional (fixed-effects) logistic regression Number of obs $=36610$ Wald chi2 $(6)=57.85$ Prob $>$ chi2 $=0.0000$

Log pseudolikelihood $=-14249.597$

Pseudo R2 $=0.0294$

(Std. Err. adjusted for clustering on countrypair)

\begin{tabular}{r|rccccc}
\hline \multirow{2}{*}{ gdppc } & Odds Ratio & Robust \\
& Std. Err. & $z$ & P>|z| & [95\% Conf. Interval] \\
\hline popi & .9874308 & .0056103 & -2.23 & 0.026 & .9764958 & .9984882 \\
popj & 1.056068 & .0095501 & 6.03 & 0.000 & 1.037515 & 1.074953 \\
gdpcapi & .8927966 & .0232083 & -4.36 & 0.000 & .8484485 & .9394626 \\
gdpcapj & 1.146728 & .0331554 & 4.74 & 0.000 & 1.083551 & 1.213588 \\
up3 & .9720182 & .0253684 & -1.09 & 0.277 & .9235474 & 1.023033 \\
down3 & 1.000253 & .0186385 & 0.01 & 0.989 & .9643815 & 1.037459 \\
\hline
\end{tabular}

Conditional (fixed-eftects) logistic regress $\begin{array}{lll}\text { Number of obs } & =35204 \\ \text { Wald } \operatorname{chi}(6) & =59.4\end{array}$ Prob > chi2 $=0.0000$

Log pseudolikelihood $=-13681.847$

Pseudo R2 $=0.0331$

(Std. Err. adjusted for clustering on countrypair)

\begin{tabular}{|c|c|c|c|c|c|c|}
\hline gdppc & Odds Ratio & $\begin{array}{l}\text { Robust } \\
\text { Std. Err. }\end{array}$ & $z$ & $P>|z|$ & [95\% Conf. & Interval] \\
\hline popi & .9834117 & .0055856 & -2.95 & 0.003 & .9725249 & .9944203 \\
\hline popj & 1.058787 & .0099507 & 6.08 & 0.000 & 1.039462 & 1.07847 \\
\hline gdpcapi & .8789243 & .0241244 & -4.70 & 0.000 & .8328906 & .9275023 \\
\hline gdpcapj & 1.123957 & .0322182 & 4.08 & 0.000 & 1.062552 & 1.188911 \\
\hline up 4 & 1.458502 & .4318344 & 1.27 & 0.202 & .8163619 & 2.605742 \\
\hline down 4 & 2.276048 & 1.127661 & 1.66 & 0.097 & .8618988 & 6.010445 \\
\hline
\end{tabular}
Number of obs $=3838$ wald $\operatorname{chi} 2(6)=61.25$ Prob $>$ chi2 $=0.000$

Log pseudolikelihood $=-14958.114$

Pseudo R2 $=0.0304$

(Std. Err. adjusted for clustering on countrypair)

\begin{tabular}{r|rrrrrr}
\hline & \multicolumn{7}{|c}{$\begin{array}{c}\text { Robust } \\
\text { gdppc }\end{array}$} & Odds Ratio & Std. Err. & $z$ & P>|z| & [95\% Conf. Interval] \\
\hline popi & .9865281 & .0055303 & -2.42 & 0.016 & .9757483 & .997427 \\
popj & 1.05946 & .0104241 & 5.87 & 0.000 & 1.039225 & 1.080089 \\
gdpcapi & .89857 & .0225967 & -4.25 & 0.000 & .8553552 & .9439683 \\
gdpcapj & 1.146217 & .0321012 & 4.87 & 0.000 & 1.084995 & 1.210893 \\
up5 & .9777312 & .0137247 & -1.60 & 0.109 & .9511979 & 1.005005 \\
down5 & .9661533 & .0305523 & -1.09 & 0.276 & .9080899 & 1.027929 \\
\hline
\end{tabular}
Wald chi2(6) $=56.7$

Prob $>$ chi2 $=0.0000$

Log pseudolikelihood $=-14949.264$

Pseudo R2 $=0.0310$

(Std. Err. adjusted for clustering on countrypair)

\begin{tabular}{|c|c|c|c|c|c|c|}
\hline gdppc & Odds Ratio & $\begin{array}{l}\text { Robust } \\
\text { Std. Err. }\end{array}$ & $\mathrm{z}$ & $P>|z|$ & [95: Conf. & Interval] \\
\hline popi & .9853156 & .0055463 & -2.63 & 0.009 & .9745049 & .9962463 \\
\hline popj & 1.059912 & .0105252 & 5.86 & 0.000 & 1.039482 & 1.080743 \\
\hline gdpcapi & .8920033 & .0227868 & -4.47 & 0.000 & .8484416 & .9378016 \\
\hline gdpcapj & 1.151779 & .0337643 & 4.82 & 0.000 & 1.087467 & 1.219894 \\
\hline up 6 & .9833471 & .0209464 & -0.79 & 0.430 & .9431382 & 1.02527 \\
\hline down 6 & 1.055995 & .036825 & 1.56 & 0.118 & .9862303 & 1.130694 \\
\hline
\end{tabular}




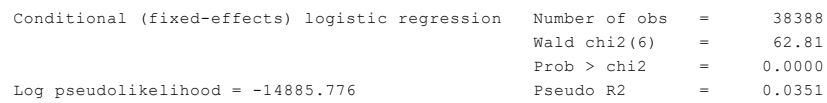

(Std. Err. adjusted for clustering on countrypair)

\begin{tabular}{|c|c|c|c|c|c|c|}
\hline gdppc & Odds Ratio & $\begin{array}{l}\text { Robust } \\
\text { Std. Err. }\end{array}$ & z & $P>|z|$ & [95\% Conf. & Interval] \\
\hline popi & .9849946 & .0055574 & -2.68 & 0.007 & .9741623 & .9959474 \\
\hline popj & 1.06058 & .010646 & 5.86 & 0.000 & 1.039919 & 1.081653 \\
\hline gdpcapi & .8825071 & .0226165 & -4.88 & 0.000 & .8392745 & .9279668 \\
\hline gdpcapj & 1.168773 & .0338189 & 5.39 & 0.000 & 1.104334 & 1.236972 \\
\hline up 7 & 1.002105 & .014753 & 0.14 & 0.886 & .9736027 & 1.031441 \\
\hline down 7 & 1.043376 & .0249593 & 1.78 & 0.076 & .9955862 & 1.093461 \\
\hline
\end{tabular}

Conditional (fixed-effects) logistic regression Number of obs $=38388$ Wald $\operatorname{chi2}(6)=62.7$ Prob $>$ chi2 $=0.000$

Log pseudolikelihood $=-14909.547$

Pseudo R2 $=0.0336$

(Std. Err. adjusted for clustering on countrypair)

\begin{tabular}{|c|c|c|c|c|c|c|}
\hline gdppc & Odds Ratio & $\begin{array}{l}\text { Robust } \\
\text { Std. Err. }\end{array}$ & $z$ & $P>|z|$ & [95: Conf. & Interval] \\
\hline popi & .9848235 & .0055605 & -2.71 & 0.007 & .9739852 & .9957823 \\
\hline popj & 1.06071 & .0106691 & 5.86 & 0.000 & 1.040004 & 1.081828 \\
\hline gdpcapi & .8842375 & .0227185 & -4.79 & 0.000 & .8408126 & .9299051 \\
\hline gdpcapj & 1.161698 & .0336262 & 5.18 & 0.000 & 1.097627 & 1.22951 \\
\hline up 8 & 1.0462 & .0394512 & 1.20 & 0.231 & .9716655 & 1.126452 \\
\hline down8 & 1.106246 & .0491596 & 2.27 & 0.023 & 1.013972 & 1.206917 \\
\hline
\end{tabular}

Conditional (fixed-effects) logistic regression Number of obs $=37961$ Wald $\operatorname{chi2}(6)=56.7$ Prob $>$ chi2 $=0.000$

Log pseudolikelihood $=-14804.937$

Pseudo R2 $=0.0292$

(Std. Err. adjusted for clustering on countrypair)

\begin{tabular}{|c|c|c|c|c|c|c|}
\hline gdppc & Odds Ratio & $\begin{array}{l}\text { Robust } \\
\text { Std. Err. }\end{array}$ & $\mathrm{z}$ & $P>|z|$ & [95\% Conf. & Interval] \\
\hline popi & .9863684 & .0055803 & -2.43 & 0.015 & .9754916 & .9973664 \\
\hline popj & 1.058403 & .0100374 & 5.99 & 0.000 & 1.038911 & 1.07826 \\
\hline gdpcapi & .8941807 & .0223419 & -4.48 & 0.000 & .8514464 & .9390599 \\
\hline gdpcapj & 1.142166 & .0316591 & 4.80 & 0.000 & 1.081771 & 1.205933 \\
\hline up 9 & .9873964 & .0171348 & -0.73 & 0.465 & .9543775 & 1.021558 \\
\hline down 9 & .9933998 & .0222507 & -0.30 & 0.767 & .9507326 & 1.037982 \\
\hline
\end{tabular}

